\title{
Camera Calibration by Hybrid Hopfield Network and Self- Adaptive Genetic Algorithm
}

\author{
Wen-jiang Xiang ${ }^{1,2}$, Zhi-xiong Zhou ${ }^{2}$, Dong-yuan $\mathrm{Ge}^{3}$, Qing-ying Zhang ${ }^{4}$ and Qing-he Yao ${ }^{5}$ \\ ${ }^{1}$ Department of Mechanical and Energy Engineering, Shaoyang University, Daxiang District, Shaoyang, 422004, P. R. China \\ ${ }^{2}$ College of Mechanical and Vehicle Engineering, Hunan University, Yuelu District, Changsha, 410082, P. R. China \\ ${ }^{3}$ School of Mechanical and Automotive Engineering, South China University of Technology, Tianhe District, Guangzhou, \\ 510640, P. R. China \\ ${ }^{4}$ Department of Production Engineering, School of Industrial Engineering and Management, Royal Institute of Technology \\ (KTH), S-100 44, Stockholm, Sweden \\ ${ }^{5}$ Department of Mechanical Engineering, Faculty of Engineering, Kyushu University, 744, Motooka, Nishi-ku,
} Fukuoka 819-0395, Japan

\begin{abstract}
A new approach based on hybrid Hopfield neural network and self-adaptive genetic algorithm for camera calibration is proposed. First, a Hopfield network based on dynamics is structured according to the normal equation obtained from experiment data. The network has 11 neurons, its weights are elements of the symmetrical matrix of the normal equation and keep invariable, whose input vector is corresponding to the right term of normal equation, and its output signals are corresponding to the fitting coefficients of the camera's projection matrix. At the same time an innovative genetic algorithm is presented to get the global optimization solution, where the cross-over probability and mutation probability are tuned self-adaptively according to the evolution speed factor in longitudinal direction and the aggregation degree factor in lateral direction, respectively. When the system comes to global equilibrium state, the camera's projection matrix is estimated from the output vector of the Hopfield network, so the camera calibration is completed. Finally, the precision analysis is carried out, which demonstrates that, as opposed to the existing methods, such as Faugeras's, the proposed approach has high precision, and provides a new scheme for machine vision system and precision manufacture.
\end{abstract}

Keywords: Camera calibration, projective matrix, Hopfield neural network, self-adaptive genetic algorithm, longitudinal direction and lateral direction

\section{INTRODUCTION}

C AMERA CALIBRATION is a process of modeling the mapping between 3D objects and 2D images, which is a key step for 3D measurement with robotic vision and manufacturing inspection, for example the measuring of micro-drill [1-2]. Ma estimated the intrinsic parameters of the camera by designing two sets of three pure orthogonal translation motions, and obtained the orientations of the camera with respect to the hand frame with a set pairwise orthogonal translation motion [3]. Zhang estimated the intrinsic and extrinsic parameters via homography matrix in the light of orthogonality of rotational matrix with the homography obtained from the 3D coordinates in target block and its 2D coordinates [4]. Cai, Li and Qiao proposed a camera calibration approach using neural networks, where transformation matrices of the binocular vision system can be replaced by the stable weights and the activation function, but there is no way to get the projective matrix of camera [5]. Ge, Yao and Xiang adopted neural network with embedded orthogonal weights to achieve camera calibration, however, the lens distortions are not included [6]. Faugeras adopted a linear pin-hole model of camera, and in the light of orthogonality of rotational matrix, obtained the intrinsic(without distortion) and extrinsic parameters of camera from the projection matrix by decomposition [7]. There is a report on the calibration of camera with the Hopfield network and simulated annealing algorithm to obtain the projection matrix, however, the global search capability of the Hopfield network and annealing algorithm is not strong [8].

Based on our research of camera calibration with computational intelligence, a flexible camera calibration with the Hopfield neural network and adaptive genetic algorithm (HNNAGA) is proposed, so the camera projection matrix is estimated according to the output vector of Hopfield network.

Our paper is organized as follows: we first introduce how the camera's pin-hole model is deduced with a sequence of geometrical transformations. In Section 3, the Hopfield neural network (abbr. as HNN) is structured according to the physical model of camera, and an innovative self-adaptive genetic algorithm is introduced, where the cross-over probability and mutation probability are tuned selfadaptively according to the evolution speed factor in longitudinal direction and the aggregation degree factor in lateral direction, respectively. In Section 4, we present the calibration experiment and precision analysis. Finally, conclusions are presented in Section 5.

\section{Perspective MOdel OF CAMERA}

As can be seen from Fig.1, OUV and $O_{1} X Y$ are the image frames measured in pixel and $\mathrm{mm}$, respectively, $O_{C} X_{C} Y_{C} Z_{C}$ and $O_{w} X_{w} Y_{w} Z_{w}$ are the camera frame and the world frame measured in mm. Assume $\left(u_{i}, v_{i}, 1\right)$ to be the homogeneous coordinates of point $p_{i},\left(X_{w i}, Y_{w i}, Z_{w i}, 1\right)$ the 
homogeneous coordinates of point $\mathrm{P}_{\mathrm{i}}$ in the world frame, the transformation relation of $O U V$ and $O_{w} X_{w} Y_{w} Z_{w}$ can be described as follows [9-11]:

$$
\boldsymbol{Z}_{\boldsymbol{c} i}\left[\begin{array}{l}
u_{\boldsymbol{i}} \\
v_{\boldsymbol{i}} \\
1
\end{array}\right]=\left[\begin{array}{llll}
\mathrm{m}_{11} & \mathrm{~m}_{12} & \mathrm{~m}_{13} & \mathrm{~m}_{14} \\
\mathrm{~m}_{21} & \mathrm{~m}_{22} & \mathrm{~m}_{23} & \mathrm{~m}_{24} \\
\mathrm{~m}_{31} & \mathrm{~m}_{32} & \mathrm{~m}_{33} & \mathrm{~m}_{34}
\end{array}\right]\left[\begin{array}{l}
X_{w i} \\
Y_{w i} \\
Z_{w i} \\
1
\end{array}\right]
$$

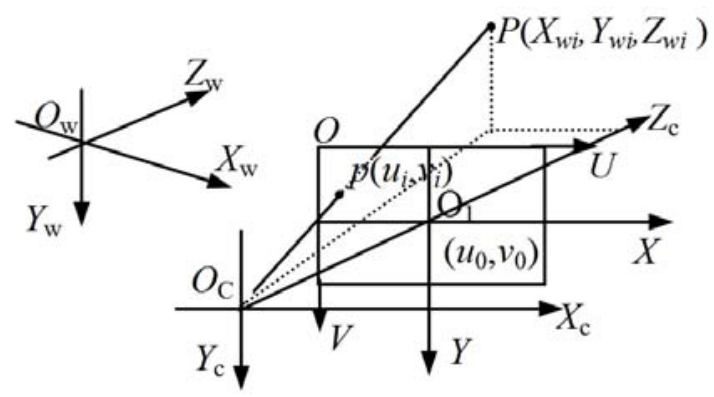

Fig.1. Perspective model of camera

With the eliminating of $Z_{c i}$ in Eq. (1), we have:

$$
\begin{aligned}
& \boldsymbol{f}_{2 i-1} \boldsymbol{v}=m_{34} u_{i} \\
& \boldsymbol{f}_{2 i} \boldsymbol{v}=m_{34} v_{i}
\end{aligned}, \quad i=1,2, \cdots, n
$$

where $\boldsymbol{f}_{2 i-1}=\left[\begin{array}{lllllllllll}X_{i} & Y_{i} & Z_{i} & 1 & 0 & 0 & 0 & 0 & -X_{i} u_{i} & -Y_{i} u_{i} & -Z_{i} u_{i}\end{array}\right]$, $\boldsymbol{f}_{2 i}=\left[\begin{array}{lllllllllllll}0 & 0 & 0 & 0 & X_{i} & Y_{i} & Z_{i} & 1 & -X_{i} u_{i} & -Y_{i} u_{i} & -Z_{i} u_{i}\end{array}\right] \quad$, $\boldsymbol{v}=\left[m_{11}, \cdots, m_{14}, m_{21}, \cdots, m_{24}, m_{31}, \cdots, m_{33}\right]^{T}$, If $n$ points' coordinates in $O U V$ and $O_{W} X_{W} Y_{W} Z_{W}$ are tested, an overdetermined equation is obtained and described as follows

$$
\boldsymbol{F v}=\boldsymbol{e}
$$

where $\boldsymbol{F}$ is $2 n \times 11$ matrix, consisting of row vectors such as $\boldsymbol{f}_{1}, \boldsymbol{f}_{2}, \cdots, \boldsymbol{f}_{2 i-1}, \boldsymbol{f}_{2 i}, \cdots, \boldsymbol{f}_{2 n}, \quad$ and $\boldsymbol{e}=\left[\begin{array}{llllllll}u_{1} & v_{1} & u_{2} & v_{2} & \cdots & \cdots & u_{n} & v_{n}\end{array}\right]^{T}$ is column vector. Thus, the normal equation can be obtained by pseudo-inverse means as follows:

$$
F^{T} F v=I
$$

where $\boldsymbol{F}^{\boldsymbol{T}} \boldsymbol{F}$ is a symmetric matrix, whose elements are $a_{i j}$ ( $i=1, \cdots, 11, j=1, \cdots, 11$ ), and $\boldsymbol{I}=\boldsymbol{F}^{T} \boldsymbol{e}$. Let $\boldsymbol{A}=\boldsymbol{F}^{\boldsymbol{T}} \boldsymbol{F}$, thus we have

$$
A v=I
$$

\section{CAMERA CALIBRATION WITH HYBRID HOPFIELD NETWORK AND INNOVATIVE GENETIC ALGORITHM}

\subsection{Design of THE Hopfield neural network}

While the camera calibration is carried out, a continuous Hopfield neural network based on dynamics is adopted. Its structure is shown in Fig.2, whose inputs are elements of vector $\boldsymbol{I}$ in Eq.(5), the weights of the network keep invariable during the iteration, which are the elements of symmetric matrix $\boldsymbol{A}$ in Eq.(5), and state variables $v_{1}(t), v_{2}(t), \cdots, v_{11}(t)$ are elements of vector $\boldsymbol{v}$ in Eq.(5). Thus, the pin-hole model of camera is transformed to the form of Hopfield network. As for the $\underline{i}^{\text {th }}$ neuron, $u_{i}$ is the input signal of $\underline{i}^{\text {th }}$ neuron, which is composed of two groups: external input $-I_{i}$, and $\sum_{j=1}^{11} a_{i j} v_{j}$, i.e., the feedback links with other neurons, where $a_{i j}$ is a corresponding element of matrix $\boldsymbol{A}$ in Eq. (5); $R_{i}$ and $C_{i}$ are the input resistor and capacitor[12-14].

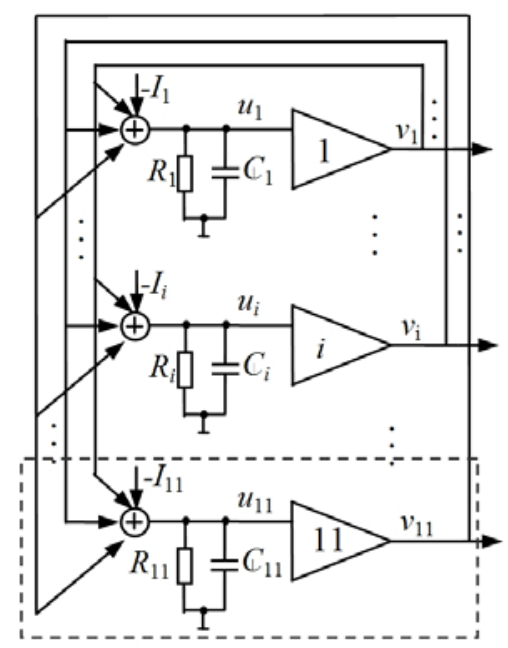

Fig.2. Structure of Hopfield network

According to Kirchhoff's current law, the relation of $i^{\text {th }}$ neuron's input and output is

$$
C_{i} \frac{d u_{i}}{d t}=\sum_{j=1}^{11} a_{i j} v_{j}-I_{i}-\frac{u_{i}}{R_{i}}, \quad i=1,2, \cdots, 11
$$

The network's activation function is the hyperbolic tangent function, that is

$$
v_{i}=g\left(u_{i}\right)=S_{i} \frac{1-e^{-\lambda_{i} u_{i}}}{1+e^{-\lambda_{i} u_{i}}}
$$

where $\lambda_{i}$ is curve's slope at the principle point.

At the same time, we define energy function as follows

$$
E_{N}=-\frac{1}{2} \sum_{i=1}^{11} \sum_{j=1}^{11} a_{i j} v_{j} v_{i}+\sum_{i=1}^{11} \frac{1}{R_{i}} \int_{0}^{v_{i}} g_{i}^{-1}(v) d v+\sum_{i=1}^{11} I_{i} v_{i}
$$


where $g^{-1}\left(v_{i}\right)$ is inverse function of $g\left(u_{i}\right)$, because $\boldsymbol{A}$ is a symmetrical matrix, thus

$$
\frac{d E_{N}}{d t}=\sum_{i=1}^{11} \frac{\partial E_{N}}{\partial v_{i}} \cdot \frac{d v_{i}}{d t}=-\sum_{i=1}^{11} C_{i} \frac{d g^{-1}\left(v_{i}\right)}{d v_{i}}\left(\frac{d v_{i}}{d t}\right)^{2}
$$

Because $C_{i}>0, v_{i}=g\left(u_{i}\right)$ and its inverse function $g^{-1}\left(v_{i}\right)$ is monotonously ascending, so $d E_{N} / d t \leq 0$ and the network will come to equilibrium along with iterations. Assuming that the $R_{i}$ is infinite, thus the third term in right of Eq.(6) can be viewed as zero, so Eq.(6) can be viewed as Eq.(5) while $C_{i}\left(d u_{i} / d t\right)=0$. If the point is the global optimum, the output signals $v_{1}, v_{2}, \cdots, v_{11}$ of Hopfield network are taken as elements of fitted projection matrix in Eq.(5).

According to Eq.(6), its iteration algorithm is obtained as follows,

$$
u_{i}=R_{i}\left(\sum_{j=1}^{11}\left(a_{i j} v_{j}-I_{i}\right)\left(1-\exp \left(-t / R_{i} C_{i}\right)\right)\right)+u_{i-1} \exp \left(-t / R_{i} C_{i}\right)
$$

where $t$ is the integral time.

\subsection{Self-adaptive genetic algorithm}

Genetic algorithm is stochastic search mechanics, which consists of reproduction, cross-over and mutation. In the solving program, an individual consist of 11 variables, which are the output signals $v_{1}, v_{2}, \cdots, v_{11}$ of Hopfield network, and the real code is adopted to obtain the individuals at random [15]. If two individuals are denoted as vector $\boldsymbol{i}(t)$ and $\boldsymbol{j}(t)$ at $\mathrm{t}^{\text {th }}$ generation, their distance is $H_{t}\left(\boldsymbol{x}_{i}\right)=\|\mathbf{i}(t)-\mathbf{j}(t)\|_{2}$, which is used to describe the nonsimilarity among individuals, or the non-similarity of the individuals between parent generation and offspring generation. In the experiment, according to the knowledge of camera model's intrinsic and extrinsic parameters, and the experiment circumstances, the search space of every individual is $(-20000,20000)$; at the same time lets the colony size be $M=80$, and the iteration timer is 300 . In the algorithm, cross-over probability and mutation probability are adjusted according to the characteristic in longitudinal direction and lateral direction.

The operation of cross-over consists of taking two individuals among the current population and in partially exchanging their respective information, obtaining two individuals who replace them. We adopt the arithmetic cross-over that produces two complimentary linear combinations of the parents:

$$
\left\{\begin{array}{l}
\mathbf{i}(t)=r \mathbf{i}(t)+(1-r) \mathbf{j}(t) \\
\mathbf{j}(t)=(1-r) \mathbf{i}(t)+r \mathbf{j}(t)
\end{array}\right.
$$

where $r$ is a random number between 1 and 0 .

Mutation operation serves for introducing a noise into the information of an individual to guarantee a certain variety of the population. The non-uniform mutation randomly selects one variable of the vector $\boldsymbol{i}, \boldsymbol{j}$, and sets it equal to a nonuniform random number:

$$
x_{n}(t)= \begin{cases}x_{n}(t-1)+\left(b_{i}-x_{n}(t-1)\right) f(t), & 0 \leq \lambda_{1}<0.5 \\ x_{n}(t-1)-\left(x_{n}(t-1)-a_{i}\right) f(t), & 0.5<\lambda_{1} \leq 1\end{cases}
$$

where $x_{n}(t), x_{n}(t-1)$ are the $\mathrm{n}^{\text {th }}$ variables of individual vector $\mathbf{i}(t), a_{i}$ and $b_{i}$ are the lower and upper bound of $x_{n}(t) ; f(t)=\lambda_{2}\left(1-\frac{t}{T}\right)^{b}, t$ denotes the current generation number, $T$ is the maximum number of generations, $\lambda_{1}$ and $\lambda_{2}$ are random numbers between 1 and $0 ; b=2$, is a shape parameter and determines the nonuniform degree of the operation. It is observed that $f(t)$ monotonically tends to 0 as $t$ approaches $T$ [16].

1. Evolution speed factor

In longitudinal direction, the evolution's direction and degree of genetic algorithm are forecasted by the individual evolution speed factor. Assuming an individual is denoted as vector $\boldsymbol{x}_{i}(t)$ and $\boldsymbol{x}_{i}(t-1)$ at $(\mathrm{t}-1)^{\mathrm{th}}$ generation and $\mathrm{t}^{\text {th }}$ generation, respectively, their distance is $H_{t}\left(x_{i}\right)=\left\|x_{i}(t-1)-x_{i}(t)\right\|_{2}$. If we let the interval of two generations as the sample unit time, the distance $H_{t}\left(x_{i}\right)$ can be taken as evolution speed for an individual. In general, the distance of past two generations is larger than the next two generations'. In the paper, let $H_{t}(x)$ be the average distance, that is $H_{t}(x)=\frac{1}{M} \sum_{i=1}^{M} H_{t}\left(x_{i}\right)$, at the same time drawing on the experience of the normalization method, the evolution speed factor is written as

$$
e_{t}=\frac{H_{t}(x)}{\max \left(H_{1}(x), H_{2}(x), \cdots, H_{t}(x)\right)}
$$

Thus $0<e_{t} \leq 1$, the larger the $e_{t}$, the faster the evolution speed; while $e_{t}$ approximate 0 , the algorithm stagnates or the optimal solution is achieved.

\section{Aggregation degree factor}

In lateral direction, if the individual's diversity decreases too soon during the iteration, the algorithm may not find the global optimization solution for the system. In order to represent the individual's diversity, the aggregation degree factor is introduced. Assuming all individuals' central position coordinate vector is $\boldsymbol{x}$ in the $\mathrm{t}^{\text {th }}$ generation, the sum distance of all individuals between $\boldsymbol{x}$ is $d_{t}=\sum_{i=1}^{M}\left\|\boldsymbol{x}_{i}-\boldsymbol{x}\right\|_{2}$, so the aggregation degree factor is given by the following equation:

$$
\sigma_{t}=\frac{d_{t}(x)}{\max \left(d_{1}(x), d_{2}(x), \cdots, d_{t}(x)\right)}
$$


It is obvious that $0<\sigma_{t} \leq 1$. The bigger the $\sigma_{t}$ is, the more diverse the individual is, and the individuals' disturbance is more dispersed.

\subsection{Self-adaptive algorithm of cross-over probability and mutation probability}

If the evolution speed of individuals is high, the algorithm can search optimization solution at a large scope. On the other hand, if the aggregation degree factor of individuals is small, the algorithm will trap into local optimization easily [17-18], so we propose an innovative approach to obtain the global optimization solution. According to the characteristic of the individual's motion trajectory from the lateral direction and longitudinal direction, at the beginning, crossover probability $p_{\mathrm{c}}$ and mutation probability $p_{\mathrm{m}}$ should increase along with the increasing of gathering of individuals, and increase with decreasing of individual evolution speed accordingly. On the other hand, to guarantee convergence of algorithm, the cross-over probability $p_{\mathrm{c}}$ and mutation probability $p_{\mathrm{m}}$ should adopt a smaller value for individuals with higher fitness degree. The $p_{\mathrm{c}}$ and $p_{\mathrm{m}}$ are modified dynamically as follows,

$$
\begin{aligned}
& p_{c}=p_{\text {cini }}-\omega_{e c} \times\left(e_{t}-0.25\right)^{2}-\omega_{o c} \times\left(\sigma_{t}-0.25\right)^{2} \\
& p_{m}=p_{\text {mini }}-\omega_{e m} \times\left(e_{t}-0.4\right)^{2}-\omega_{o m} \times\left(\sigma_{t}-0.45\right)^{2}
\end{aligned}
$$

where $\omega_{e c}, \omega_{e m}, \omega_{\sigma c}$ and $\omega_{\sigma m}$ are the coefficients of evolution speed factor and aggregation degree factor; their ranges are defined as $0<\omega_{e c}, \omega_{e m}<1,0<\omega_{\sigma c}, \omega_{\text {om }}<1$. In the experiment, let $p_{\text {cini }}=0.7, p_{\text {mini }}=0.1$, which are initial values, and keep invariant in iteration. And let $\omega_{e c}=0.4$, $\omega_{e m}=0.2, \omega_{\sigma c}=0.1$ and $\omega_{o m}=0.75$. Self-adaptive tunings of cross-over probability $p_{\mathrm{c}}$ and mutation probability $p_{\mathrm{m}}$ are shown in Fig.3, where $\mathrm{AD}$ factor denotes aggregation degree factor, and ES factor denotes evolution speed factor.

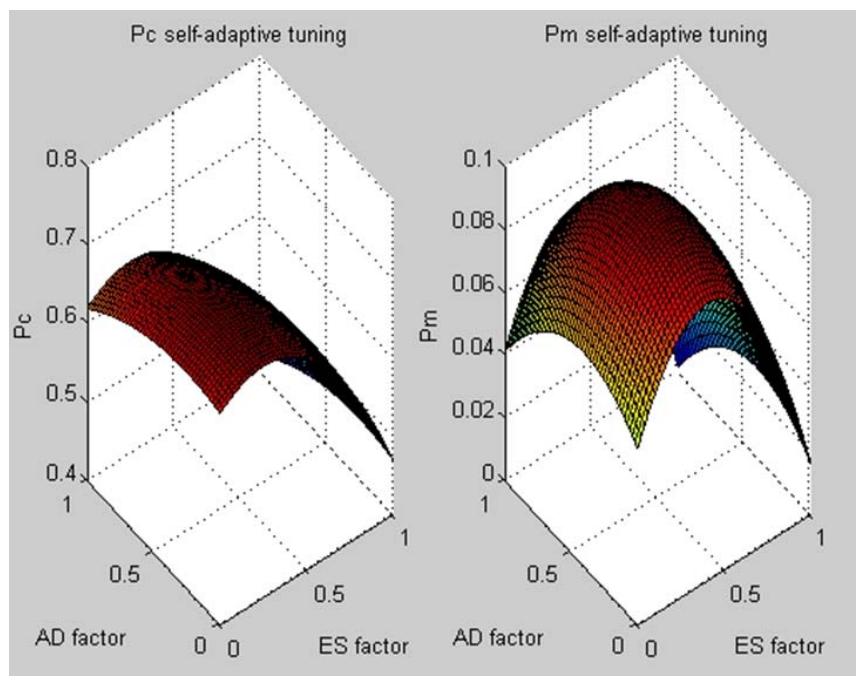

Fig.3. Self-adaptive tuning of $p_{\mathrm{c}}$ and $p_{\mathrm{m}}$

\subsection{Flowchart of THE calibration program}

The flow chart of the program is illustrated in Fig.4. In the calibration program, let $R=100000, C=1, t=0.0001$, $s=1, \lambda=0.001$. Because the energy function is negative, as can be seen from Eq.(8), so in the iteration algorithm, in order to made the motion trajectory come to the least Lyapunov function, let

$u_{i}=-R_{i}\left(\sum_{j=1}^{11}\left(a_{i j} v_{j}-I_{i}\right)\left(1-\exp \left(-t / R_{i} C_{i}\right)\right)\right)+u_{i-1} \exp \left(-t / R_{i} C_{i}\right)$

The Hopfield neural network is trained 5 times for each individual in every generation. $1 /\left(E-1 \times 10^{-12}\right)$ is taken as fitting function of genetic algorithm, where $E$ is the performance index of the Hopfield network, and the constant $1 \times 10^{-12}$ is taken as offset value to avoid the performance index of the Hopfield network to approach 0 . According to the evolution speed factor and aggregation degree factor we judge whether the system comes to the neighborhood of global optimization state (abbr. as NGOS). If not, the next vector, which is row vector of matrix $\boldsymbol{A}$, is put into the system; and if all vectors are over, next round is restarted again; else the program only runs Hopfield to obtain the precision solution. When the system comes to global equilibrium state, the outputs of the networks converge to stable values, which can be taken as the elements of camera's projection matrix, thus the camera calibration is finished.

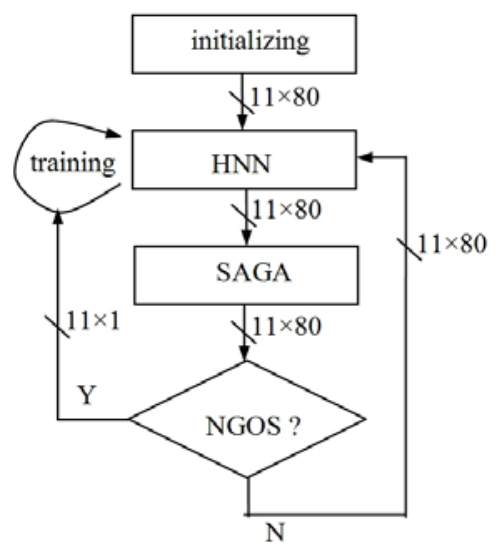

Fig.4. Flowchart of calibration program

\section{CALIBRATION EXPERIMENT AND ANALYSIS OF PRECISION}

\subsection{Calibration experiment}

The eye-hand system of the robot consists of 4 free degree manipulators, camera, target block, servo control system and so on, which is shown in Fig.5. In the camera calibration experiment, firstly the 3D coordinates of the feature points are measured relative to a selected world coordinate system. Then the images of target block are taken with the camera, and the feature extraction is carried out, so the edge information of blind holes is obtained using a technique based on edge-detection, which is shown in Fig.6. Finally, the 2D coordinates of blind holes' center in the image planes are estimated with sub-pixel accuracy [19-20]. 


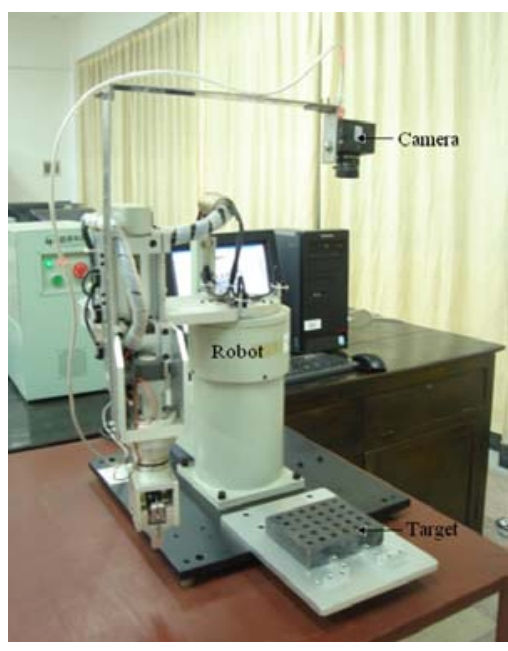

Fig.5. Vision system and manipulator
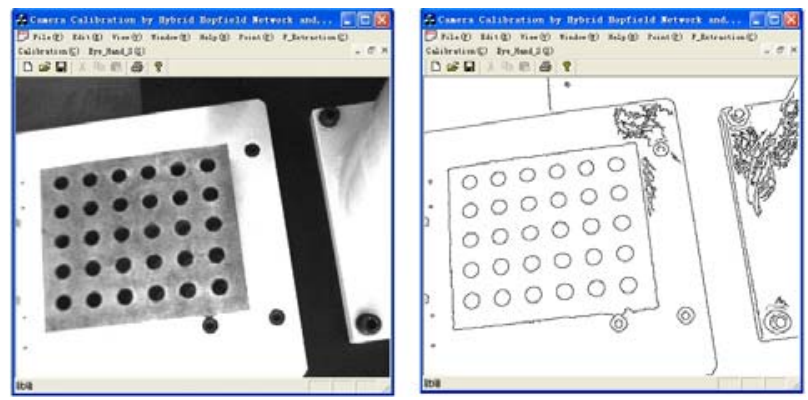

Fig.6. Sampled calibration block image

In the camera calibration program, the improved genetic algorithm drives the individuals to come to the neighborhood of the global optimization state, when the Hopfield network comes to the global equilibrium state and the stable output vector is obtained, from which the projection matrix of camera is estimated and shown in Table 1.

Table 1. Camera projection matrix obtained from the outputs of Hopfield network

\begin{tabular}{cccc}
\hline $2.3147 \times 10^{1}$ & -1.0659 & 8.2205 & $-2.0448 \times 10^{2}$ \\
$8.8249 \times 10^{-1}$ & $2.3592 \times 10^{1}$ & 9.6617 & $5.9399 \times 10^{2}$ \\
$-9.5297 \times 10^{-4}$ & $5.0855 \times 10^{-4}$ & $2.7898 \times 10^{-2}$ & 1 \\
\hline
\end{tabular}

\subsection{Analysis of precision}

There is no unified precision index standard for a machine vision system, in the precision analysis experiment, let image residual errors be taken as calibration precision. First, the projected points' coordinates in the image plane are estimated according to the projection matrix and 3D coordinates of sampled points in world frame, then image errors between estimated coordinates and actual tested coordinates are taken as calibration precision of camera, which can be expressed as follows.

$$
\delta_{u}=\sqrt{\left(u_{i}-u\right)^{2}+\left(v_{i}-v\right)^{2}}
$$

If the coordinates of projected points in the image plane are estimated according to the projection matrix and 3D coordinates, we have

$u_{i}=\left(m_{11} X_{w i}+m_{12} Y_{w i}+m_{13} Z_{w i}+m_{14}\right) /\left(m_{31} X_{w i}+m_{32} Y_{w i}+m_{33} Z_{w i}+m_{34}\right)$

$v_{i}=\left(m_{21} X_{w i}+m_{22} Y_{w i}+m_{23} Z_{w i}+m_{24}\right) /\left(m_{31} X_{w i}+m_{32} Y_{w i}+m_{33} Z_{w i}+m_{34}\right)$

So the coordinates of projected points $\left(u_{i}, v_{i}\right)$ in the image plane are obtained.

While the calibration precision of camera is carried out, we move the manipulator to have the target block at some integral number positions, such as $450.000 \mathrm{~mm}$ and 410.00 $\mathrm{mm}$ in $\mathrm{Z}$ axis. 6 feature points are chosen at random, and the coordinates of their center in the world frame are measured, which are shown in Table 2. Then the actual tested 2D coordinates of blind hole center are obtained from the image of the target block, which are shown in the $1^{\text {st }}$ line of Table 3. Then according to the results of camera calibration with the proposed approach, the coordinates of feature points projected in image planes are estimated according to Eqs.(18) and (19), which are obtained and shown in the $2^{\text {nd }}$ line of Table 3.

Finally, according to equation (17), the precision indices for the proposed technique are shown in the $3^{\text {rd }}$ line of Table 3.

If the camera calibration method, such as the Faugeras approach, is adopted, where the least square method (abbr. as LSM) is used to carry out data processing, so the projection matrix of camera is obtained and shown in Table 4.

Table 2. 3D coordinates of sampled points (/mm)

\begin{tabular}{ccc}
\hline No. 1 & No. 2 & No. 3 \\
\hline$(46.0005,25.0068,450.0000)$ & $(126.0098,65.0108,450.0000)$ & $(66.0047,105.0050,450.0000)$ \\
\hline No. 4 & No. 5 & No. 6 \\
\hline$(46.0109,45.0090,410.0000)$ & $(126.0073,85.0081,410.0000)$ & $(126.0084,105.0063,410.0000)$ \\
\hline
\end{tabular}


Table 3. Coordinates of actual and solved, and precision index (/pixel)

\begin{tabular}{|c|c|c|c|}
\hline & No. 1 & No. 2 & No. 3 \\
\hline Tested coordinates & $(335.1021,411.9745)$ & $(470.8410,489.0109)$ & $(362.4592,551.9580)$ \\
\hline $\begin{array}{c}\text { Coordinates estimated by } \\
\text { HNNAGA }\end{array}$ & $(335.1915,412.0574)$ & $(470.9315,489.0908)$ & $(362.5461,552.0439)$ \\
\hline $\begin{array}{c}\text { Image errors by } \\
\text { HNNAGA }\end{array}$ & 0.1219 & 0.1208 & 0.1222 \\
\hline $\begin{array}{c}\text { Coordinates estimated by } \\
\text { LSM }\end{array}$ & (335.2093, 412.0735) & (470.9493, 489.1098) & $(362.5644,552.0618)$ \\
\hline \multirow[t]{2}{*}{ Image errors by LSM } & 0.1459 & 0.1467 & 0.1478 \\
\hline & No. 4 & No. 5 & No. 6 \\
\hline Tested coordinates & $(336.7771,455.5485)$ & $(484.6368,539.6602)$ & $(482.5184,577.3503)$ \\
\hline $\begin{array}{c}\text { Coordinates estimated by } \\
\text { HNNAGA }\end{array}$ & $(336.8623,455.6319)$ & $(484.7281,539.7426)$ & $(482.6087,577.4348)$ \\
\hline $\begin{array}{c}\text { Image errors by } \\
\text { HNNAGA }\end{array}$ & 0.1192 & 0.1229 & 0.1237 \\
\hline $\begin{array}{c}\text { Coordinates estimated by } \\
\text { LSM }\end{array}$ & $(336.8810,455.6483)$ & $(484.7445,539.7585)$ & $(482.6259,577.4490)$ \\
\hline Image errors by LSM & 0.1441 & 0.1458 & 0.1460 \\
\hline
\end{tabular}

Table 4. Projective matrix of camera obtained by LSM

\begin{tabular}{cccc}
\hline $2.3103 \times 10^{1}$ & -1.0654 & 8.2013 & $-2.0316 \times 10^{2}$ \\
$8.8619 \times 10^{-1}$ & $2.3540 \times 10^{1}$ & 9.6391 & $5.9373 \times 10^{2}$ \\
$-9.4046 \times 10^{-4}$ & $5.0298 \times 10^{-4}$ & $2.7834 \times 10^{-2}$ & 1 \\
\hline
\end{tabular}

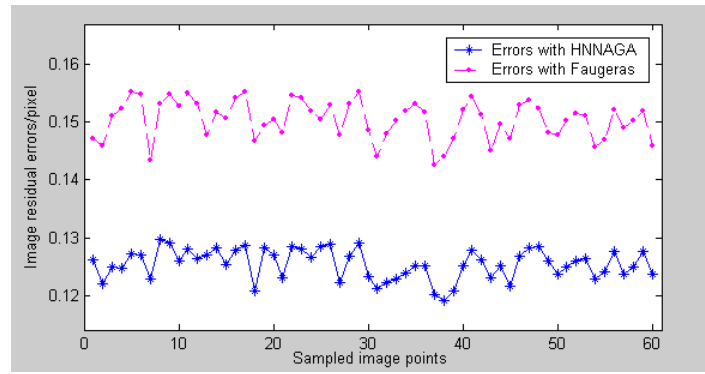

Fig.7. Residual errors of image with two approaches

At the same time, in order to obtain the precision indices for the least square method, the $2 \mathrm{D}$ coordinates of space points projected on the image plane are estimated according to Eqs.(18) and (19) and shown in the $4^{\text {th }}$ line of Table 3; so the precision indices of calibration, i.e. the image residual errors are obtained and shown in the $5^{\text {th }}$ line of Table 3 .

On the other hand, when the target block is located at two positions, such as $450.00 \mathrm{~mm}$ and $410 \mathrm{~mm}$, the other 56 feature point coordinates besides the above 6 points are obtained too; and the precision indices are obtained for two calibration approaches, then all the precision indices, i.e. image residual errors, are plotted in one figure and shown in Fig.7.
As can be seen from Fig.7, the residual errors between the tested image coordinates and estimated coordinates are demonstrated and composed with two approaches. The maximum error and minimum image error are 0.1297 (pixel) and 0.1192 (pixel) with the proposed approach, respectively; and the maximum and minimum of the image residual errors are 0.1554 (pixel) and 0.1426 (pixel) with Faugeras's; and the average residual errors of the proposed approach and the least square method are 0.1254 (pixel) and 0.1504 (pixel), respectively.

\section{CONCLUSIONS AND FURTHER WORK}

A novel approach for camera calibration based on hybrid Hopfield network and self-adaptive genetic algorithm is proposed in this paper. As opposed to other techniques, our main contributions are [3-7]:

Firstly, the Hopfield neural network is structured according to the normal equation derived from experiment data and physical model of camera, so the fitting algorithm of camera calibration is transformed into dynamic neural network. The energy function of Hopfield network always decreases along with its iteration. The projection matrix of camera is obtained from stable output vectors of the Hopfield network in the experiment when the global optimization solution is obtained. Second, an innovative genetic algorithm is proposed to get the global optimization solution, where the trajectory of individual and aggregation degree of colony are described with evolution speed factor and aggregation degree factor from longitudinal direction and lateral direction, respectively, so the cross-over probability $p_{\mathrm{c}}$ and mutation probability $p_{\mathrm{m}}$ are tuned dynamically according to the evolution speed factor and the aggregation degree factor. 
The proposed approach possesses merits of simple computation and high accuracy, which makes the system meet the precise requirement for intelligent test and precision manufacture.

The future work will focus to achieve the measurement of micro-drill's defects, such as rounded corner, main lips' chips, long-short edge, overlap(or gap), non-concentricity and so on with machine vision system and the proposed technique.

\section{ACKNOWLEDGMENT}

The work described in this paper is partially supported by National Natural Science Foundation of China (51075133), Hunan Provincial Natural Science Foundation of China (09JJ6092), and Scientific Research Fund of Hunan Provincial Education Department (09B092), and the Hunan Province Universities Innovative Platform Open Fund Project (11K059). The authors also gratefully acknowledge the helpful suggestions of the reviewers, which have improved the presentation.

\section{REFERENCES}

[1] Chen, J.Y., Lee, B.Y., Lee, K.C., Chen, Z.K. (2010). Development and implementation of a simplified tool measuring system. Measurement Science Review, 10 (4), 142-146.

[2] Ge, D.Y., Yao, X.F., Xiang, W.J. (2011). Application of BP neural network and genetic algorithm in testing of micro-drill's rounded corner. Sensor Letters, 9 (5), 1943-1947.

[3] Ma, S.D. (1996). A Self-calibration technique for active vision system. IEEE Transactions on Robotics and Automation, 12 (1), 114-120.

[4] Zhang, Z. (2000). A flexible new technique for camera calibration. IEEE Transactions on Pattern Analysis and Machine Intelligence, 22 (11), 1330-1334.

[5] Cai, S., Li, Q.A., Qiao, Y.F. (2007). Camera calibration of attitude measurement system based on BP neural network. Journal of Optoelectronics - Laser, 18 (7), 832-834. (in Chinese)

[6] Ge, D.Y., Yao, X.F., Xiang, W.J. (2011). Application of neural network with embedded orthogonal weight on calibration of camera's intrinsic and extrinsic parameters. Optics and Precision Engineering, 19 (11), 2782-2790. (in Chinese)

[7] Faugeras, O.D., Toscani, G. (1986). The calibration problem for stereo. In IEEE Computer Society Conference on Computer Vision and Pattern Recognition, 22-26 June 1986. IEEE Computer Society, 15-20.

[8] Ge, D.Y., Yao, X.F., Yu, M.Q. (2010). Research on Hopfield network \& simulated annealing in camera calibration. ICIC Express Letters, 4 (4), 1257-1262.
[9] Zhao, Z.X., Wen, G.J., Zhang, X., Li, D.R. (2012). Model-based estimation for pose, velocity of projectile from stereo linear array image. Measurement Science Review, 12 (3), 104-110.

[10] Wang, Y.Q., Gao, H., Liang, Y. (2011). Parametric detection of rice kernel shape using machine vision. Sensor Letters, 9 (3), 1212-1219.

[11] Ulises, P., Cho, S., Asfour and Shihab. (2009). Volumetric calibration of stereo camera in visual servo based robot control. International Journal of Advanced Robotic Systems, 6 (1), 35-42.

[12] Wang, W.L., Wu, Q.D., Xu, X.L. (2002). Hopfield neural network approach for job-shop scheduling problems. Acta Automatica Sinica, 28 (5), 838-844.

[13] Li, Y., Tang, Z., Xia, G.P., Wang, R.L. (2005). A positively self-feedbacked Hopfield neural network architecture for crossbar switching. IEEE Transactions on Circuits and Systems I: Regular Papers, 52 (1), 200-206.

[14] Pajares, G. (2006). A Hopfield neural network for image change detection. IEEE Transactions on Neural Networks, 17 (5), 1250-1264.

[15] Chen Z.Q., Wang, R.L. (2011). Two efficient realcoded genetic algorithms for real parameter optimization. International Journal of Innovative Computing, Information and Control, 7 (8), 48714884.

[16] Song, X.D., Liu, X.B. (2010). ETL tasks matching and scheduling based on genetic algorithm. ICIC Express Letters, 4 (4), 1245-1250.

[17] Zhang, X.P., Du, Y.P., Qin, G.Q., Qin, Z. (2005). Adaptive particle swarm algorithm with dynamically changing inertia weight. Jounal of Xi'an Jiaotong University, 39 (10), 1039-1042. (in Chinese)

[18] Xu, W.F., Li, C., Liang, B., Liu, Y., Xu, Y.S. (2008). The Cartesian path planning of free floating space robot using particle swarm optimization. International Journal of Advanced Robotic Systems, 6 (1), 35-42.

[19] Xu, G., Li, X.T., Su, J., Chen, R., Liu, J.F. (2012). A method for the estimation of the square size in the chessboard image using gray-level co-occurrence matrix. Measurement Science Review, 12 (2), 68-73.

[20] Lin, C.J., Wang, J.G., Chen, S.M. (2011). 2D/3D face recognition using neural network based on hybrid taguchi-particle swarm optimization. International Journal of Innovative Computing, Information and Control, 7 (2), 537-553. 